\title{
Particle Creation in a Big-bung Universe
}

Although the 'big-bang' models of the Universe were formulated nearly fifty years ago, investigations close to the big-bang events have been few and far between. Physicists have, on the whole, been hesitant about applying their laws in conditions very different from those that operate 'here' and 'now'. One notable exception is the work of George Gamow more than twenty-five years ago about element synthesis during the first few seconds after the big bang. This led to the prediction of the microwave background which was first observed in 1965 .

During the few years since this important discovery, cosmologists have become bolder in tackling the big bang. Many authors, like Zeldovich, Sexl, Parker and others, have examined the possibility of particle creation as a gravitational effect in the highly curved and rapidly expanding Universe just after the big bang. An interesting and novel attempt in this direction has recently been made by Audretsch of the Fachbereich Physik der Universität, Konstanz (Nuovo Cim., B17, 284; 1973). This work arose partly out of the author's discussions with the relativity groups in London. Audretsch's approach may be briefly described as follows.

In quantum mechanics the propagation of massive spin- $\frac{1}{2}$ particles, like the electron, is described by the Dirac equation. In the flat space of special relativity, such a particle of given mass and momentum can exist in one of four possible states, described by a plane wave. Each state is characterised with spin, which can be $+\frac{1}{2}$ or $-\frac{1}{2}$ and by energy which can be positive or negative (but with the same magnitude). In order to avoid the awkward negative energy solutions Dirac proposed the 'sea' of negative energy particles. This sea is completely filled and is unobservable. A free particle of positive energy can propagate for ever without taking any notice of this sea. If, however, there is an external force acting on it, such as the electromagnetic field, it changes energy. This can lead to a particle from the sea 'jumping up', for example. Such a particle, and the 'hole' it has created in the sea, then appear as a pair of particles. This is how particle creation from an energy source like radiation is believed to take place.

Audretsch has applied this picture to the expanding Universe and he comes up with certain important differences. When he considers the plane wave solution of the Dirac equation in the expanding Universe, he finds that only in certain special circumstances do the positive and negative energy solutions stay unmixed. The special cases are (1) the particle in question has no mass (a neutrino, for example) and (2) if the particle is massive it is at rest in the cosmological substratum. For a massive moving particle mixing of states must take place. That is if the particle is initially described by a plane wave with positive energy, it will later on also acquire states of negative energy. This curious effect, Audretsch finds, is entirely due to the expansion of the Universe. By analogy with the flat space picture just described, he concludes that the appearance of negative energy states must be interpreted as particle creation. This is how matter appears in the expanding Universe.

According to Audretsch the effect of expansion is significant for particle creation when the age of the Universe is comparable to the microphysical time scale-when the Universe is only about $10^{-21} \mathrm{~s}$ old! This is hardly surprising in a theory which seeks to relate microphysics with cosmology. In this work Audretsch has steered clear of many relevant but complex issues, such as the implications of quantum field theory (this work does not quantitise the Dirac field), the effects of quantitising the space-time geometry, the implications of the negative energy sea for Einstein's field equations and so on. No doubt such problems will keep theoretical bigbang cosmologists busy in years to come.

J. V. N.

\section{DEMOGRAPHY}

\section{Conflicting Population Reports}

\section{from our Demography Correspondent}

ONE of the professed aims of the United Nations World Population Year, 1974, is improvement of knowledge of population trends, particularly in the Third World. The recent history of population projections for the United Kingdom, where population statistics are among the world's best, in terms of range and quality, indicates that this will not be easy and the conflicting findings of two recently published studies give no basis for supposing that the situation is improving in Britain.

A set of official projections (Population Projections, No. 3, 1972-2012, HMSO, London; 1973) suggests a population of just under 58 million for England and Wales by the year 2011, a rise of about 9 million over present numbers. By contrast Gilje and Gould of the Greater London Council Intelligence Unit ( $Q$. Bull., No. 24; September 1973) suggest a possible population of about 50 million for the same date, which is only a fractional increase over the present figure. The team of GLC researchers has a very good record of population studies and the projection methods of the two reports are broadly similar. How then can such conflicting reports result?
The answer is that the assumptions on future fertility patterns differ crucially. Both reports use the same mortality and migration assumptions and, though some demographers might argue the future mortality decline assumptions are optimistic, changes in this factor during the projection period are likely to be minimal.

Fertility patterns result from a complex of economic and social factors affecting the decisions of individuals not merely on one matter but severalprincipally age at marriage, desired family size and the spacing of births in relation to marriage and preceding births. To predict future changes in each of these elements demands the prediction of future patterns of economic and social factors which influence them. This is the familiar problem of an intricate set of interdependencies and feedback relationships. Economists and resource planners see population estimates as basic inputs for their studies of future trends but demographers equally require these projections to make their fertility assumptions.

The population increase suggested in the official projections stems from the assumption that mean completed family 